\title{
Multisystem inflammatory syndrome in children during the COVID-19 pandemic in Turkey: first report from the Eastern Mediterranean
}

\author{
Yasemin Ozsurekci ${ }^{1}$ - Sibel Gürlevik ${ }^{1}$. Selman Kesici ${ }^{2}$ Ummusen Kaya Akca ${ }^{3} \cdot$ Pembe Derin Oygar $^{1} \cdot$ Kubra Aykac $^{4}$. \\ Dilek Karacanoglu $^{2}$ - Ozlem Sarıtas Nakip ${ }^{2} \cdot$ Sare Ilbay $^{1} \cdot$ Ban Katlan $^{2}$. Illker Ertugrul ${ }^{5}$ - Ali Bülent Cengiz ${ }^{1}$. \\ Ozge Basaran ${ }^{3} \cdot$ Burcu Ceylan Cura Yayla $^{4} \cdot$ Jale Karakaya $^{6} \cdot$ Yelda Bilginer $^{3} \cdot$ Benan Bayrakci $^{2} \cdot$ Mehmet Ceyhan $^{1}$. \\ Seza Ozen ${ }^{3}$
}

Received: 2 January 2021 / Revised: 31 January 2021 / Accepted: 2 February 2021 / Published online: 12 February 2021

(C) International League of Associations for Rheumatology (ILAR) 2021, corrected publication 2021

\begin{abstract}
Objective We aimed to describe the typical clinical and laboratory features and treatment of children diagnosed with multisystem inflammatory syndrome in children (MIS-C) and to understand the differences as compared to severe/critical pediatric cases with COVID-19 in an eastern Mediterranean country.

Methods Children (aged $<18$ years) who diagnosed with MIS-C and severe/critical pediatric cases with COVID-19 and were admitted to hospital between March 26 and November 3, 2020 were enrolled in the study.

Results A total of 52 patients, 22 patients diagnosed with COVID-19 with severe/critical disease course and 30 patients diagnosed with MIS-C, were included in the study. Although severe COVID-19 cases and cases with MIS-C share many clinical and laboratory features, MIS-C cases had longer fever duration and higher rate of the existence of rash, conjunctival injection, peripheral edema, abdominal pain, altered mental status, and myalgia than in severe cases $(p<0.001$ for each). Of all, $53.3 \%$ of MIS-C cases had the evidence of myocardial involvement as compared to severe cases (27.2\%). Additionally, C-reactive protein (CRP) and white blood cell (WBC) are the independent predictors for the diagnosis of MIS-C, particularly in the existence of conjunctival injection and rash. Corticosteroids, intravenous immunoglobulin (IVIG), and biologic immunomodulatory treatments were mainly used in MIS-C cases rather than cases with severe disease course. There were only three deaths among 52 patients, one of whom had Burkitt lymphoma and the two cases with severe COVID-19 of late referral.

Conclusion Differences between clinical presentations, acute phase responses, organ involvements, and management strategies indicate that MIS-C might be a distinct immunopathogenic disease as compared to pediatric COVID-19. Conjunctival injection and higher CRP and low WBC count are reliable diagnostic parameters for MIS-C cases.
\end{abstract}

\section{Key Points}

- MIS-C cases had longer fever duration and higher rate of the existence of rash, conjunctival injection, peripheral edema, abdominal pain, altered mental status, and myalgia than in severe/critical pediatric cases with COVID-19.

- Higher CRP and low total WBC count are the independent predictors for the diagnosis of MIS-C.

- MIS-C might be a distinct immunopathogenic disease as compared to pediatric COVID-19.

Seza Ozen

sezaozen@gmail.com

1 Faculty of Medicine, Department of Pediatric Infectious Diseases, Hacettepe University, Ankara, Turkey

2 Faculty of Medicine, Department of Pediatrics, Division of Pediatric Intensive Care Unit, Hacettepe University, Ankara, Turkey

3 Faculty of Medicine, Department of Pediatrics, Division of Pediatric Rheumatology, Hacettepe University, Ankara, Turkey
4 Department of Pediatric Infectious Diseases, University of Health Science Ankara Training and Research Hospital, Ankara, Turkey

5 Division of Cardiology, Department of Pediatrics, Faculty of Medicine, Hacettepe University, Ankara, Turkey

6 Faculty of Medicine, Department of Biostatistics, Hacettepe University, Ankara, Turkey 
Keywords Kawasaki disease $\cdot$ Pediatrics $\cdot$ Hyperinflammation $\cdot$ Multisystem inflammatory syndrome in children (MIS-C) · Multisystem inflammatory syndrome in adults (MIS-A)

\section{Introduction}

The emerging infection due to Severe Acute Respiratory Syndrome Coronavirus 2 (SARS-CoV-2), named COVID19 , commonly has led a mild upper respiratory illness in children [1-4]. For instance, a study of 220 children with confirmed COVID-19 from Turkey, including our hospital, reported that $66 \%$ of cases presented with an asymptomatic and mild disease course, whereas $2.7 \%$ of the pediatric cases had a severe disease [5]. In the beginning of May 2020, a report of eight pediatric cases with a severe hyperinflammation as well as the involvement of several organs was published from London [6]. Subsequently increasing number of cases were reported globally, and the entity consists of children with the temporal association with COVID-19 who manifested with persistent fever, rash, conjunctivitis, and symptoms of organ involvement such as cardiovascular and gastrointestinal system was later called as multisystem inflammatory syndrome in children (MIS-C) [7, 8]. The diagnosis of MIS-C cases, based on six principal elements including clinical characteristics and the results of laboratory examinations, as defined by World Health Organization (WHO) and Centers for Disease Control and Prevention (CDC) [7, 8] as follows: (a) pediatric age, (b) persistence of fever, (c) elevated inflammatory biomarkers, (d) signs and/or symptoms of organ dysfunction, (e) lacking an alternative plausible diagnosis, and (f) evidence of COVID-19 or COVID-19 exposure.

One of the challenges of us was to differentiate children with MIS-C and Kawasaki disease (KD) as reported from many centers $[9,10]$ because of the possibility of an association between KD and positive testing for SARS-CoV-2 [11]. In our hospital, 129 pediatric cases with $\mathrm{KD}$ were diagnosed between 2007 and 2019, and approximately, a 3.7-fold increase in the incidence of KD cases was observed in the beginning of pandemic [12]. Subsequently, this fold-increase had a steep rise. Another challenge for us was to understand the actual nature of COVID-19 cases with a severe/critical disease course who had a really higher cytokine/chemokine response as compared to children with milder disease course (unpublished data). During the pandemic, because Hacettepe University is one of the main tertiary care centers, serving a large geographic area in Turkey, mainly severe COVID-19 cases have been referred from other pediatric hospitals. These severe COVID-19 cases with an excessive hyperinflammation, which have revealed with the same frequency throughout the pandemic, have many overlap features with MIS-C.
Therefore, we evaluated and compared the demographics, clinical, laboratory and imaging findings, and management strategies of the children aged under 18 years diagnosed with severe COVID-19 disease and met the criteria for MIS-C according to $\mathrm{WHO}$ and $\mathrm{CDC}[7,8]$.

\section{Materials and methods}

We recruited pediatric patients aged under 18 years, who were confirmed as COVID-19 with a severe/critical disease course and MIS-C to this cohort between March 26 and November 3, 2020, and who were admitted to Hacettepe University Faculty of Medicine and University of Health Sciences, Ankara Educating and Training Hospital. The clinical features of children with COVID-19 with severe/critical disease course included in this cohort have been previously approved by the institutional review board at the Hacettepe University following informed consent from parents/guardians. Inclusion of patients with MIS-C was approved by the institutional review board at the University of Health Sciences, Ankara Educating and Training Hospital. All parents/guardians provided written informed consent.

In this study, the severity of pediatric COVID-19 cases was based on the clinical characteristics and the results of laboratory examinations and radiologic imaging, as defined by Dong et al. [13]. Asymptomatic cases were defined as those with a positive test result without any clinical or radiological findings; mild and moderate cases were defined as the existence of the symptoms of respiratory tract infection without or with pneumonia, respectively. Severe disease was defined as with progressive respiratory disease, dyspnea, and central cyanosis, and critically ill disease course was defined as those who presented with acute respiratory distress syndrome or respiratory failure, shock, and organ dysfunction. The children were included who met the CDC [7] and/or WHO definitions [8] for MIS-C. Those definitions are fundamentally based on a couple of principle elements as follows: (a) pediatric age, (b) persistent fever, (c) presence of inflammatory biomarkers, (d) findings of organ dysfunction, (e) absence of any other diagnosis, and (e) temporal association with COVID-19. Additionally, all patients in our cohort required proof of SARS-CoV-2 exposure via PCR analysis and/or serology to exclude possible cases who have Kawasaki disease rather than a new emerging condition associated with COVID-19. Additionally, we excluded the cases who are diagnosed with an infection caused by another viral or bacterial agent such as 
adenovirus, Epstein-Barr virus, or Streptococci spp., which may share similar features.

The variables potentially associated with the COVID-19 infection and MIS-C included age; sex; clinical presentation; the duration of symptoms; the findings of organ involvement; laboratory and radiological findings such as white blood cell count (WBC), absolute leukocyte and neutrophil counts, acute phase reactants including C-reactive protein (CRP) and sedimentation rate, full biochemical analyses including renal and liver function tests, electrolytes, albumin, coagulation tests, ferritin and posteroanterior chest X-ray, abdominal ultrasonography, and chest computed tomography (CT) if needed; previous medical history; underlying diseases (i.e., heart disease, chronic lung disease, developmental delay, hematological disease, and tracheostomy); use of medical devices (mechanical ventilation, extracorporeal membrane oxygenation [ECMO], and etc.); treatment modalities such as antiviral, antimicrobial therapies, immunosuppressive, and immunomodulatory treatments; intensive care unit (ICU)/pediatric ICU (PICU) admission; and outcome. Data regarding the demographic and clinical characteristics of patients were collected via patients' charts, computerized administrative, pharmacy, and laboratory databases of the hospitals.

We diagnosed suspected cases, according to our national COVID-19 guidelines. Suspected cases with positive reverse transcriptase-polymerase chain reaction (RT-PCR) or serumspecific antibodies against 2019-nCoV were accepted as confirmed cases [14]. Euroimmun anti-SARS-Co-V2 ELISA Ig $\mathrm{G}$ and Colloidal Gold rapid test were used for detection of anti-SARS-CoV-2 Ig G.

\section{Statistical analysis}

All statistical tests were calculated using SPSS 22.0 for Windows (IBM, Chicago, Ill). Descriptive statistics were used to summarize the primary characteristics of patients, including the median and minimum-maximum for continuous variables. Categorical variables are presented as per frequencies and in percentages and were compared using the chi-square test. Continuous variables are presented as median (min-max), and differences in continuous variables between the groups were tested by using the Mann-Whitney $U$-test. Receiver-operating characteristic (ROC) curve and area under the ROC curve (AUC) of the levels of the laboratory parameters were estimated for the patients. After ROC analysis, the best cut-off point was determined by using Youden Index for CRP and WBC. The sensitivity and specificity of these parameters was calculated according to the best cut off point. Moreover, a decision tree was created with the CART (classification and regression tree) method to find the discriminative clinical and laboratory parameters, and the validity of the model was examined with the leave one out cross-validation technique. Also, multiple logistic regression analysis was used to identify the risk factors. $P$ value of less than 0.05 was considered statistically significant.

Hacettepe University Ethics Board and University of Health Sciences, Ankara Educating and Training Hospital for Non-Interventional Studies reviewed and approved the study protocol.

\section{Results}

\section{Evidence of SARS-CoV-2 infection and clinical features}

A total of 52 patients were included in the study: Over a period from March 26 to November 3, 2020, 22 patients diagnosed with COVID-19 with severe/critical disease course and from August 2 to November 3, 2020, 30 patients diagnosed with MIS-C according to criteria of WHO $(n=29)$ and CDC $(n=1)$ (Fig. 1). Demographic data and clinical features are summarized in Table 1. Median age of severe COVID-19 cases was 12 years, and MIS-C cases was 9 years with no significant difference. There was no any gender predominance in the groups, and more than $80 \%$ of the cases in both groups had a known contact history of a case with COVID-19. The history of contact with a COVID-19 case was detected $86.7 \%$ in MIS-C cases and $81.8 \%$ in severe COVID-19 cases. Of all, four $(14.3 \%)$ of the MIS-C cases had positive PCR results for SARS-CoV-2, and three of them had simultaneously positive PCR and serology results.

Approximately $60 \%$ of the severe COVID-19 cases had an underlying disease, which was most commonly neurometabolic/genetic disorders, followed by hematologic/ oncologic and chronic pulmonary diseases, whereas $83.3 \%$ of the MIS-C cases had no comorbidities. Almost all cases had fever, which was the most common clinical feature in both groups. Whereas the duration of fever was mainly less than 3 days in severe cases (90.5\%), a total of $86.7 \%$ of the MIS-C cases had fever of at least 4 days. The clinical findings, such as rashes, bilateral conjunctival injection, peripheral extremity edema, chest pain, myalgia, the changes of mental status, and abdominal pain were significantly higher in MIS-C patients as compared to severe/critical cases ( $p<0.001$ for each). One MIS-C case underwent emergency operation for suspected appendicitis that was ultimately diagnosed as mesenteric lymphadenitis before admission to our center, and two cases were followed up with a suspicion of appendicitis in our center. Additionally, tachycardia was significantly higher in MIS-C cases $(p=0.03)$, whereas cough was significantly higher in cases with severe/critical disease course $(p<0.001)$.

\section{Imaging and laboratory findings}

Of the 30 MIS-C patients who underwent chest imaging (radiography or computed tomography), 21 (70\%) had ground 
glass opacities, local patchy shadowing, interstitial abnormalities, and pleural effusion. More than $30 \%$ of the cases $(n=10)$ had pleural effusion on chest tomography. Although no coronary artery abnormalities and aneurysms were identified in MIS-C cases, coronary artery abnormalities were detected in only one COVID-19 case with severe disease course. Echocardiography detected the evidence of myocardial involvement in $53.3 \%(n=16)$ of MIS-C cases and in $27.2 \%$ $(n=6)$ of the severe COVID-19 cases (Table 1).

There were differences in the laboratory parameters between the patients with MIS-C and those with severe COVID-19 (Fig. 2). The median white blood cell (WBC), C-reactive protein (CRP), D-dimer, ferritin, albumin, and sodium levels of MIS-C cases $(13,900 / \mu \mathrm{L}$ [min-max, 4,200$45,000], 19.6 \mathrm{mg} / \mathrm{dl}$ [min-max, 1.2-35.6], $4.1 \mathrm{mg} / \mathrm{L}$ [min$\max , 1.0-80.0], 456.5 \mathrm{ng} / \mathrm{ml}$ [min-max, 44-3846], $2.6 \mathrm{~g} / \mathrm{dl}$ [min-max, 2.1-3.9], and $133 \mathrm{mEq} / \mathrm{L}$ [min-max, 125-154], respectively) were different than the COVID-19 children with a severe disease course $(7,200 / \mu \mathrm{L}$ [min-max, $1,300-15,800]$, $1.2 \mathrm{mg} / \mathrm{dl}$ [min-max, 0.01-12.3], $0.9 \mathrm{mg} / \mathrm{L}$ [min-max, 0.335.2 ], $127 \mathrm{ng} / \mathrm{ml}$ [min-max, 14-3,762], $3.6 \mathrm{~g} / \mathrm{dl}$ [min-max, 2.1-4.9], and $138 \mathrm{mEq} / \mathrm{L}$ [min-max, 130-152], respectively) $(p=<0.001, p=<0.001, p=0.003, p=0.03, p=0.003$, and $p$ $=0.002$, respectively). The troponin and B-type natriuretic peptide (BNP) levels of MIS-C cases were $58 \mu \mathrm{g} / \mathrm{L}$ (min$\max , 2.6-9,248)$ and $872 \mathrm{ng} / \mathrm{L}(\min -\max , 0.08-5704)$, respectively. The troponin and BNP levels were determined for only 13 COVID-19 cases with severe disease course, and the levels were $6.4 \mu \mathrm{g} / \mathrm{L}$ (min-max, $0.1-14,153.6$ ), and $10 \mathrm{ng} /$ L (min-max, 10-2,682.6), respectively. Despite of the low patient count in severe group, the difference between the troponin and BNP levels was significantly different $(p=$ 0.02 and $p=<0.001$, respectively) and the data not shown for troponin and BNP.

Although the difference was not significant between the groups $(p=0.09)$, the absolute lymphocyte counts (ALC) was slightly lower in MIS-C cases $(975 / \mu \mathrm{L}$ [min-max, 230-7,300]) as compared to severe COVID19 cases $(1,640 / \mu \mathrm{L}$ [min-max, 70-7,800]); whereas, the absolute neutrophil counts (ANC) was significantly higher in MIS-C cases $(10,900 / \mu \mathrm{L}$ [min-max, 2,550-35,500]) as compared to severe COVID-19 cases $(4,040 / \mu \mathrm{L}$ [min$\max , 0-14,500])(p=<0.001)$.

We further analyzed whether the laboratory parameters could be used as diagnostic predictors for MIS-C cases. The results of the MIS-C cases showed that area under the ROC curve of CRP was determined significant $(p<0.001)$ and area under curve (AUC) of the ROC curve was 0.951 (95\%CI: 0.85-0.99) (Fig. 3). The performance of CRP as a diagnostic test was found to be good with the best cut-off point as 12.36; the sensitivity and specificity of CRP was 83.3 and $100 \%$, respectively. The AUC of the ROC curve was 0.840 for WBC (95\%CI: 0.71-0.92) $(p<0.001)$ (Fig. 3). The performance of WBC as a diagnostic test was found to be good with the best cut-off point as 11,000; the sensitivity and specificity of WBC was 73.3 and $90.9 \%$, respectively.

\section{CART decision tree and logistic regression}

Additionally, we tried to find discriminative symptoms and laboratory finding between MIS-C and severe cases. When all variables that found significant in univariate analysis were taken into

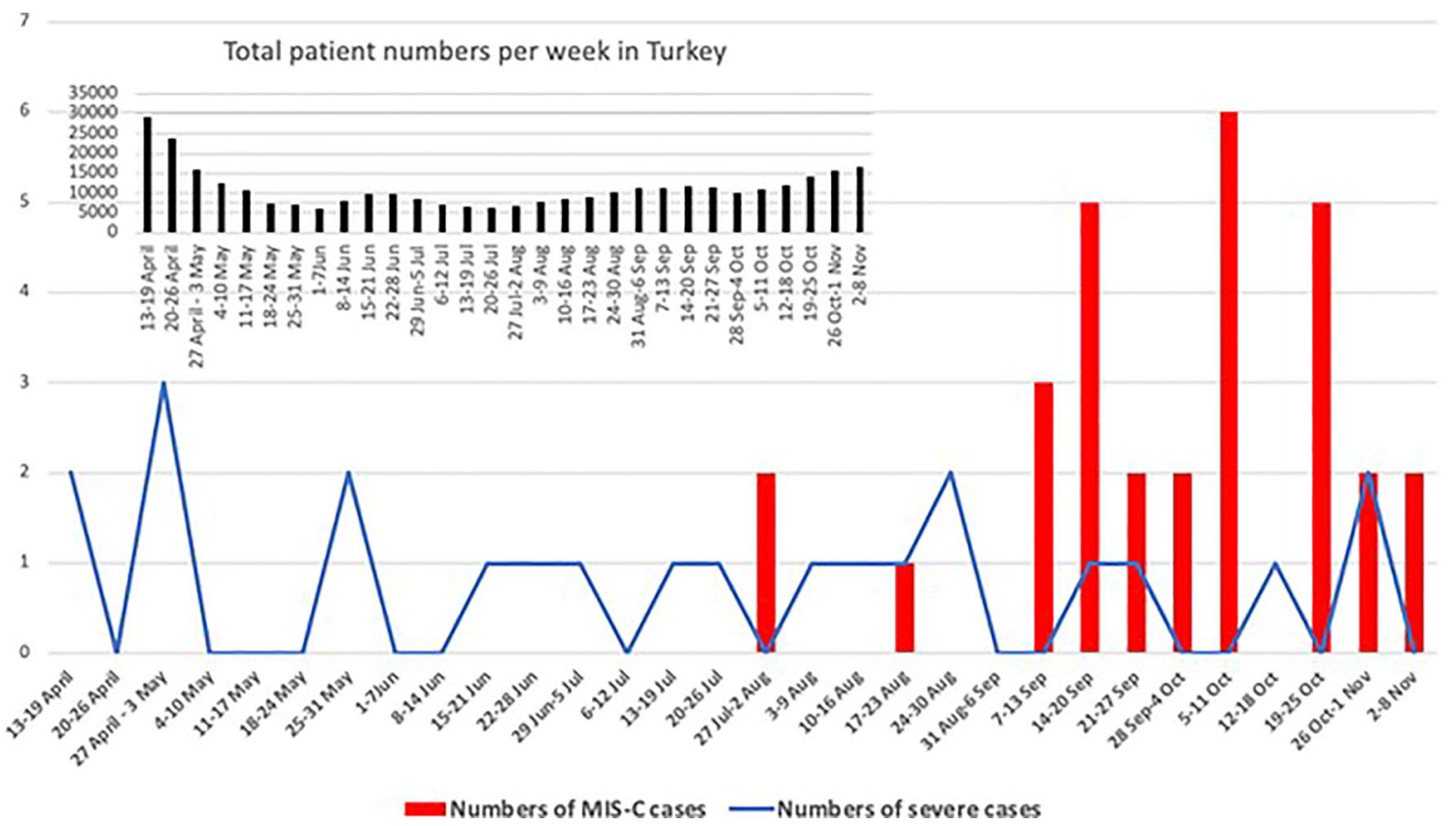

Fig. 1 Time line of the pediatric cases who presented with the diagnosis of MIS-C and severe/critical COVID-19 
Table 1 Characteristics of the study cohort

\begin{tabular}{|c|c|c|c|}
\hline Characteristics & $\begin{array}{l}\text { Cases with MIS-C } \\
(n=30)\end{array}$ & $\begin{array}{l}\text { Severe/Critical cases } \\
\text { with COVID-19 } \\
(n=22)\end{array}$ & $p^{* *}$ \\
\hline Age, years, median (min-max) & $9(1-17)$ & $12(0-17)$ & 0.56 \\
\hline Male, $n(\%)$ & $16(53.3)$ & $15(68.2)$ & 0.29 \\
\hline Known contact with a COVID-19 case $(n, \%)$ & $26(86.7)$ & $18(81.8)$ & 0.63 \\
\hline SARS-CoV-2 PCR positive $(n, \%)$ & $4(14.3)$ & $22(100)$ & $<0.001$ \\
\hline SARS-CoV-2 PCR negative $(n, \%)$ & $26(86.7)$ & 0 & NA \\
\hline \multicolumn{4}{|l|}{ SARS-CoV-2 IgG serology in PCR positive patients } \\
\hline Positive & 3 & 0 & \\
\hline Negative & 1 & 0 & \\
\hline \multicolumn{4}{|l|}{ SARS-CoV-2 IgG serology in PCR negative patients } \\
\hline Positive & $25(96.1)$ & 0 & \\
\hline Negative $^{a}$ & $1(4.2)$ & 0 & \\
\hline \multicolumn{4}{|l|}{ Underlying disease $(n, \%)$} \\
\hline None & $25(83.3)$ & $9(40.9)$ & $<0.001$ \\
\hline Underlying disease & $5(16.7)$ & $13(59.0)$ & \\
\hline Chronic pulmonary diseases & $2(6.7)$ & $2(9.1)$ & \\
\hline Neurometabolic/genetic diseases & 0 & $6(27.3)$ & \\
\hline Hematologic/oncologic diseases & 0 & $3(13.6)$ & \\
\hline Obese & $2(6.7)$ & 0 & \\
\hline Type $1 \mathrm{DM}$ & $1(3.3)$ & 0 & \\
\hline \multicolumn{4}{|l|}{ Clinical presenting features $*(n, \%)$} \\
\hline Fever & $30(100)$ & $22(100)$ & 1.0 \\
\hline \multicolumn{4}{|l|}{$(d)$, median $(\min -\max )$} \\
\hline$<3$ days & $4(13.3)$ & $20(90.5)$ & \\
\hline 4 days & $9(30)$ & $2(9.1)$ & $<0.001$ \\
\hline$>5$ days & $17(56.7)$ & 0 & \\
\hline Rash & $21(70)$ & $1(4.5)$ & $<0.001$ \\
\hline Bilateral conjunctival injections & $29(96.7)$ & $1(4.5)$ & $<0.001$ \\
\hline Peripheral extremity edema & $23(76.7)$ & $2(9.1)$ & $<0.001$ \\
\hline Chest pain & $20(76.9)$ & $5(26.3)$ & $<0.001$ \\
\hline Cough & $4(13.3)$ & $15(68.2)$ & $<0.001$ \\
\hline Respiratory distress & $21(70)$ & $17(77.3)$ & 0.56 \\
\hline Tachycardia & $29(96.7)$ & $17(77.3)$ & 0.03 \\
\hline Abdominal pain & $25(83.3)$ & $8(36.4)$ & $<0.001$ \\
\hline Vomiting & $21(70.0)$ & $8(36.4)$ & 0.02 \\
\hline Diarrhea & $11(36.7)$ & $6(27.3)$ & 0.48 \\
\hline Headache & $7(23.3)$ & $2(9.5)$ & \\
\hline Lethargy, altered mental status & $20(69)$ & $3(13.6)$ & 0.28 \\
\hline Myalgia & $22(88)$ & $7(31.8)$ & $<0.001$ \\
\hline $\begin{array}{l}\text { Organomegaly (hepatomegaly, splenomegaly, } \\
\text { or both) }\end{array}$ & $23(76.7)$ & $7(33.3)$ & 0.002 \\
\hline \multicolumn{4}{|l|}{ Radiological findings, $n(\%)$} \\
\hline Abnormal chest X-ray & $21(70.0)$ & $17(81.0)$ & 0.38 \\
\hline Abnormal chest tomography & $14(93.3)$ & $18(85.7)$ & 0.47 \\
\hline \multicolumn{4}{|l|}{ Hospitalization } \\
\hline $\begin{array}{l}\text { Hospital length of stay (days), median } \\
\text { (min-max) }\end{array}$ & $7(3-29)$ & $8(3-31)$ & 0.12 \\
\hline ICU admission $n, \%$ & $14(46.7)$ & $18(81.8)$ & 0.01 \\
\hline Length of ICU stay (days), median (min-max) & $4(1-19)$ & $5(1-25)$ & 0.07 \\
\hline
\end{tabular}


Table 1 (continued)

\begin{tabular}{|c|c|c|c|}
\hline Characteristics & $\begin{array}{l}\text { Cases with MIS-C } \\
(n=30)\end{array}$ & $\begin{array}{l}\text { Severe/Critical cases } \\
\text { with COVID-19 } \\
(n=22)\end{array}$ & $p^{* *}$ \\
\hline Respiratory support, $n(\%)$ & & & 0.018 \\
\hline None & $14(46.7)$ & $6(27.3)$ & \\
\hline Oxygen only & $7(23.3)$ & $8(36.4)$ & \\
\hline High flow support & 0 & $2(9.1)$ & \\
\hline Non-invasive ventilation & $6(20.0)$ & 0 & \\
\hline Invasive mechanical ventilation & $3(10.0)$ & $6(27.3)$ & \\
\hline Complication, $n(\%)$ & & & 0.71 \\
\hline Myocarditis & $2(6.7)$ & $3(13.6)$ & \\
\hline Perimyocarditis & $4(13.3)$ & 0 & \\
\hline Pericarditis & $4(13.3)$ & $1(4.5)$ & \\
\hline Heart Failure & $10(33.3)$ & $3(13.6)$ & \\
\hline Multiorgan failure & $4(13.3)$ & $4(18.8)$ & \\
\hline Sepsis & 0 & $1(4.5)$ & \\
\hline Coronary artery aneurysms & 0 & $1(4.5)$ & \\
\hline \multicolumn{4}{|l|}{ Treatments during admission, $n(\%)$} \\
\hline Vasoactive infusion & $13(43.3)$ & $6(27.3)$ & \\
\hline Plasma exchange & $14(46.7)$ & 0 & \\
\hline Corticosteroids (2 mg/kg/day) & $27(90)$ & $11(50)$ & \\
\hline High-dose corticosteroids ( $30 \mathrm{mg} / \mathrm{kg} /$ day) & $1(3.3)$ & 0 & \\
\hline Intravenous immunoglobulin & $30(100)$ & $7(31.8)$ & \\
\hline Biologic immunomodulation $^{\mathrm{b}}$ & $26(86.7)$ & $1(4.5)$ & \\
\hline Outcome, $n(\%)$ & & & $\mathbf{0 . 0 3}$ \\
\hline Still hospitalized & $2(6.7)$ & 0 & \\
\hline Recovered & $28(93.3)$ & $19(86.4)$ & \\
\hline Mortality & 0 & $3(13.6)$ & \\
\hline
\end{tabular}

NA not applicable.

${ }^{\text {a }}$ One case with no any PCR and serology positivity had a strong COVID-19 exposure in his family.

${ }^{\mathrm{b}}$ One patient was treated with tocilizumab and 26 with anakinra.

*Percentages are computed in complete cases, as some variables contain missing value.

** Significant values are shown as bold

account and the decision tree model was obtained, a tree was formed only with "conjunctival injection." When the decision tree was formed with the variable of conjunctival injection, the sensitivity of the model was $96.7 \%$, and the specificity was $95.5 \%$ in making a distinction between the MIS-C and severe cases, and the classification accuracy of the model was $96.2 \%$.

When the decision tree was created after extracting the conjunctivitis variable, the decision tree first branched for the "CRP," and in the second step it made a branch according to the "rash" variable. According to cut-off point as 12.36 for CRP at the first level: 25 patients with a CRP value above 12.36 were classified as MIS-C, and all of them were actually MIS-C (100\%). At the second level, when the CRP level was determined as less than 12.36 with the existence of the rash, 20 of the 21 cases were actually MIS-C (95.2\%). In this decision tree model in which two variables were used, the same result was obtained with the above model (the model with conjunctivitis).

When the multiple logistic regression model with backward elimination technique was created together with conjunctivitis and CRP, classification accuracy of the model was $100 \%$.

\section{Treatment and outcome}

Favipiravir was used for all cases in both groups without seeking a positive PCR result because of the unclear pathogenesis of MIS-C cases, to target the possible viral infection. Sixty-six percent of MIS-C cases had cardiac involvement ranging from mild to severe and $43.3 \%$ required vasopressor support and/or fluid resuscitation. Anticoagulants (low molecular weight heparin), 

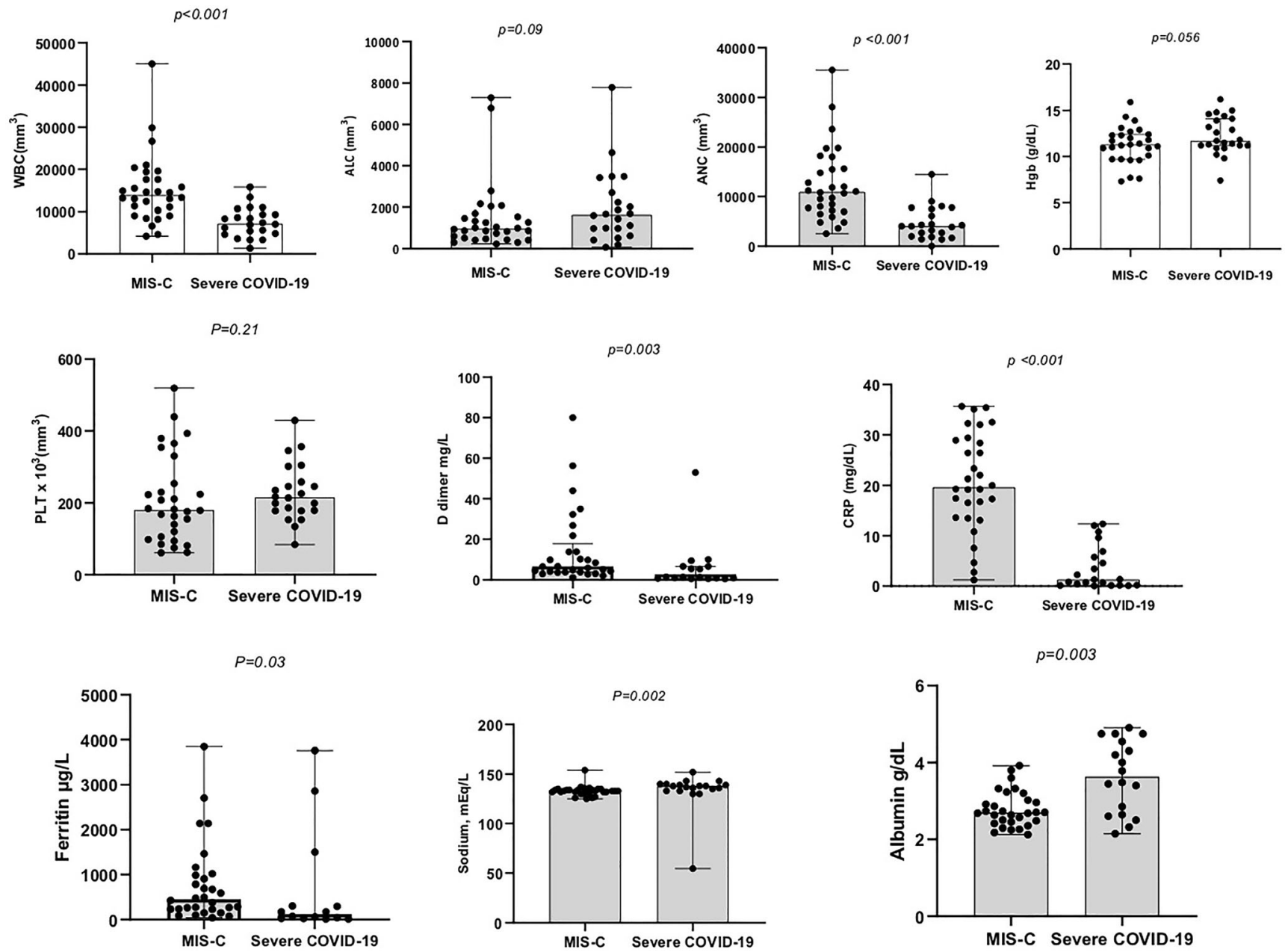

Fig. 2 The laboratory value alterations in patients with MIS-C compared with severe/critical COVID-19 cases. WBC, white blood cell, ALC, absolute leukocyte count, ANC, absolute neutrophil count, PLT, platelet, CRP, C-reactive protein

Fig. 3 The ROC curve of WBC and CRP levels on admission for MIS-C cases. WBC, white blood cell and CRP, C-reactive protein. The ROC curve of CRP was determined significant $(p<0.001)$, and area under curve (AUC) of the ROC curve was 0.951 (95\%CI: 0.85-0.99), and the AUC of the ROC curve was 0.840 for WBC (95\%CI: 0.71-0.92) $(p<0.001)$

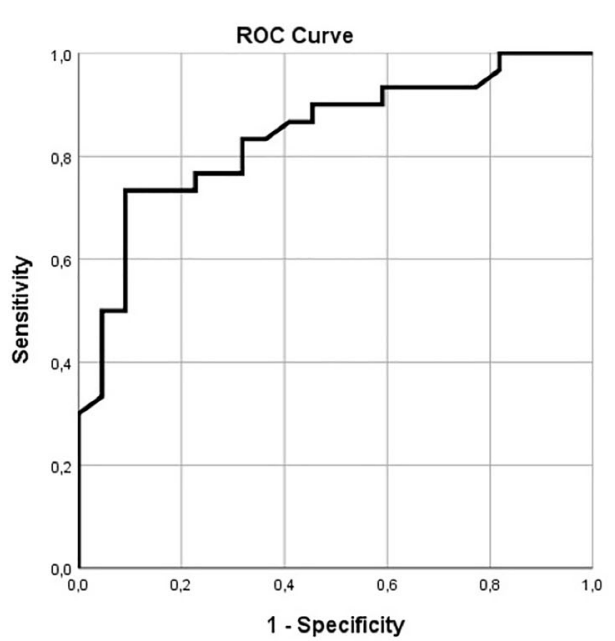

White blood cell (WBC)

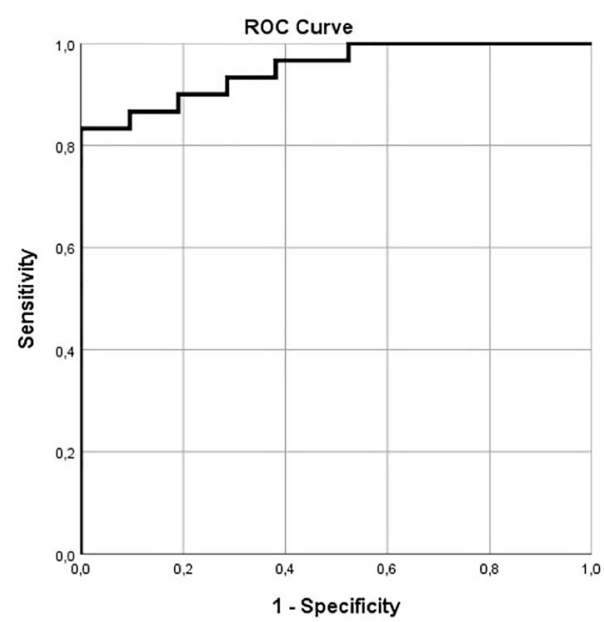

C-reactive protein (CRP) 
corticosteroids, intravenous immunoglobulin (IVIG), and biologic immunomodulatory treatments (anakinra) were mainly used in MIS-C cases rather than cases with severe disease course (Table 1). All 30 MIS-C cases received high dose IVIG $(2 \mathrm{~g} / \mathrm{kg})$, and all received concomitant corticosteroids. Only one case required second IVIG administration. Methylprednisolone was used at a dose of 2 $\mathrm{mg} / \mathrm{kg}$ for all but one, who needed bolus methylprednisolone $(30 \mathrm{mg} / \mathrm{kg}, 3$ consecutive days). Anakinra was preferred as the immunomodulatory treatment and used at a dose of $2 \mathrm{mg} / \mathrm{kg}$ /day for 23 of MIS-C cases and $4 \mathrm{mg} / \mathrm{kg} /$ day for remaining three. Of all, 26 MIS-C patient and one severe COVID-19 case treated with anakinra. The duration of anakinra use was shorter than reported in the literature. Tocilizumab was used in only one patient before referral to our hospital. The MIS-C patients received empirical broad-spectrum antibiotic treatment, and all test results for bacteria (urine and blood culture) were negative.

Pediatric intensive care unit (PICU) admission was required for $14(46.7 \%)$ of the MIS-C cases and $18(81.8 \%)$ in children with severe disease course, the difference being significant $(p=0.01)$. The length of PICU stay of MIS-C cases was 4 days, and the difference was not significant compared to the PICU stay of severe cases with COVID-19 $(p=0.07)$. Extracorporeal membrane oxygenation (ECMO) was required for two severe cases and for only one MIS-C case.

Three patients with severe COVID-19 died: two patients who died were aged 1 and 7 years. They had no preexisting comorbidities; however, they were referred late in their disease course. The remaining one, who died at the age of 14 due to respiratory failure, had Burkitt lymphoma. No deaths were recorded among the MIS-C cases.

\section{Discussion}

Here, we compared the various parameters of children with COVID-19 with severe/critically ill disease and MIS-C cases. Although there are certain clinical and laboratory similarities between the MIS-C and severe COVID-19 cases, important differences such as the presentation findings and responses of WBC and CRP in MIS-C cases were determined in the present study. We found that conjunctival injection was significantly distinctive feature between the MIS-C and severe cases and WBC and CRP levels were significantly higher in MIS-C cases, and they both are independent predictors for the diagnosis of MIS-C, particularly together with the clinical findings such as conjunctival injection and rash.

While MIS-C has overlapping features with severe COVID-19 in children, inflammatory response observed in MIS-C seems much more intense according to the findings of the present study. Distinguishing clinical characteristics found in MIS-C were age, cardiac, and gastrointestinal involvement in our cohort and some of the laboratory values might provide biologic insight to the disease or may guide the clinician in the management of MIS-C, consistent to the literature [10]. Although MIS-C cases have been compared to cases with Kawasaki diseases in many studies $[9,15]$ and they have shared many similar features, the causal factor is different in MIS-C. While increased acute phase molecules including interleukin-1 $\beta$ (IL-1 $\beta$ ), IL-6, IL-10, IL-17, interferon- $\gamma$ (IFN- $\gamma$ ), C-reactive protein (CRP), and ferritin confirm acute inflammation, raised pro-B-type natriuretic peptide (proBNP) and troponin suggest myocardial dysfunction and injury in MIS-C. Contrary to the common findings of Kawasaki, increased levels of D-dimer and low levels of platelet count indicative a procoagulant phase in MIS-C [15]. Because of this possible procoagulant process and upcoming flu season, we mainly preferred to use anticoagulation with low molecular weight heparin rather than aspirin, consistently to literature [16]. In the present study, differences between acute phase response, myocardial involvement, and the procoagulant state suggest that MIS-C is a distinct disease, compared to pediatric COVID-19. In fact, MIS-C is one of the most intense forms of inflammation. Further immunological analysis is needed for defining the exact immunopathogenic factors. Additionally, although MIS-C shares certain features of adults with COVID-19 including lymphopenia and cytokine excess, there are some immunological differences, such as high neutrophil count and the sign of $\mathrm{T}$ cell exhaustion $[15,17,18]$.

COVID-19 cases have spread throughout the world and severe cases have reached an alarming number in childhood. COVID-19 is milder in children as compared to adults; however, current reports of severe hyperinflammation associated with COVID-19 in children, which is referred as MIS-C, are of increasing importance worldwide. Those MIS-C cases with a new and potentially life-threating disease course emerged particularly after late April 2020 in many countries from Europe and America [6, 9, 10, 19-22]. However, there is no published report about MIS-C cases from the eastern Mediterranean and Middle-East, where COVID-19 has displayed a similar endemic as western Europe. The first case with COVID-19 in Turkey reported in March 11, 2020. Although our severe cases were distributed to the defined study period, MIS-C cases in the present study were commonly seen after the beginning of the August 2020. These findings have shown us that MIS-C cases are seen in the society after reaching a certain case saturation. Although the incidence of MIS-C was reported 2 per 100,000 persons younger than 21 years of age in a paper from New York State [23], it seems impossible to predict a real incidence data because of the asymptomatic cases and/or mild disease course of COVID19 particularly in children. Furthermore, a crucial concern is that children could still develop MIS-C despite an asymptomatic course of COVID-19 [24-26]. As a matter of fact, about 
$15 \%$ of the cases in this study have not described any previous disease and/or contact history of COVID-19.

Since hyperinflammation is a common process in MIS-C, prompt administration of anti-inflammatory agents, including intravenous immunoglobulin (IVIG) and corticosteroids with/ without biologic immunomodulation, consistent with the literature $[10,15]$ was the key management strategy in this patient cohort. However, our pediatric centers are tertiary care referral centers in the capital of Turkey and mean admission time of the cases after the beginning of symptoms was 5.5 days. Left ventricular dysfunction was the main cardiac feature in this case series, with no of patients having coronary artery dilatation; thus, heart seems one of the main targets of the inflammatory attack according to our findings consistent with the literature $[9,16,27]$. High levels of biomarkers associated with arteritis and coronary artery disease and antibodies that target structural proteins in heart and blood vessels are also evident in MIS-C cases [9]. Intravenous immunoglobulin treatment was used for all our MIS-C cases, with the hypothesis that activation of inhibitory Fc-receptors and prevention membrane-attack complexes via complement factors by IVIG might mitigate autoantibody-associated disease process [28]. Despite a second IVIG administration was given in 12.8$62.5 \%$ of the reported MIS-C cases [11, 29-31], the benefit from such medication still remains unclear. Only one case required second IVIG administration in our cohort.

Since MIS-C typically manifests 3-4 weeks after COVID19 , many children have positive antibodies to SARS-CoV-2 $[29,30]$. One of the main questions about MIS-C is whether possible pathogenesis might be mediated by the virus or immune system and both. Although immune-mediated pathophysiology in children who had extraordinary inflammation is discussed in a couple of paper $[9,10]$ because of the good clinical response of anti-inflammatory agents, "virus associated second-hit concept" is another hot topic on account of the existence of viral particles across a number of tissues in a child who died from MIS-C [9, 27]. Furthermore, the report about multisystem inflammatory syndrome in adults (MIS-A) is an intriguing new subject for clinicians, and Morris et al. [32] reported that since the patients described in the study had minimal infectious symptoms of respiratory tract, MIS-A might be distinguished from severe COVID-19 cases as in our study. Although the pathogenesis in not well-described as in children, the possible role of persistent infection outside the upper respiratory tract infection is discussed because of the proof of the SARS-CoV-2 in various organs such as liver, brain, kidney, and gastrointestinal tract in addition to heart $[32,33]$. Additionally, consistent with the literature [22], the lack of PCR testing of stool sample as well as tissue sampling during the study period in our center prevented us to understand the possible viral replication in targeted organs, such as gastrointestinal tract, endothelium, and myocardial tissue. In the present study, one patient with no previous COVID-19 exposure was in the active disease course of COVID-19 with the absence of antibodies, and three patients had simultaneously positive PCR and IgG serology for SARS-CoV-2; therefore, this unclear pathogenesis, perhaps mixt (immune plus virus-mediated) pathogenesis has led us prefer to use also antiviral treatment in addition to anti-inflammatory agents in our MIS-C patients. Another important question on MIS-C is why some children are vulnerable to developing the hyperinflammation, as well. All of our cases were Turkish (Caucasian), and no cases but two, of whom were overweight and had hearing loss, had underlying conditions/diseases to let us explain a possible susceptibility pattern. The actual mechanisms of MIS-C, as a result, still have remained elusive.

Pediatric patients with MIS-C in this cohort were mainly treated with a combination of IVIG, corticosteroids, and recombinant IL-1-receptor antagonist (ILRA; anakinra). Lifethreatening situations due to cytokine storm could be downregulated by corticosteroids [34]. Use of corticosteroids was found to be associated with reduced mortality in pneumonia caused by viruses such as influenza and 2019-nCoV [35, 36] and shortens the duration of hospitalization and reduce the need for mechanical ventilation without causing secondary infection and other complications in a couple studies, as well [37-39]. Because of this emerging situation in worldwide, although corticosteroids were used for $50 \%$ of pediatric cases with severe/critical disease course, we prudently started to evaluate the use of them in selected patients with severe disease course in the light of the accumulated data. Despite corticosteroids provide a general immunosuppression, the actual mechanism in MIS-C still needs to be determined. ILRA neutralizes IL-1 response associated with endothelial autoantibody- and complement-mediated damage in MIS-C [9]. There are some studies with the different anti-cytokine therapies including IL-1 and IL-6 blockade in severe cases with COVID-19 as well [40]. Nevertheless, the use of clinical judgment seems crucial for the management of the cases because of variations of the background immunomodulators.

Our study has several limitations. First, it was based on retrospective data collection from a small number of pediatric patients and management were individualized by patient rather than a standardized protocol. Since children were treated with the immunomodulatory medications according to local practice, this report does not provide evidence on effectiveness of treatment of MIS-C. Second, our data is mainly descriptive with the lack of molecular analysis, and actual inferential conclusions cannot be drawn and the underlying mechanisms of MIS-C cannot be addressed from it. However, we attempted to exclude the cases who met the criteria of Kawasaki disease (KD) without any evidence of COVID-19. Kawasaki disease is one of the most prominent manifestations of acute inflammatory response in children. Thus, it is not surprising that the hyperinflammation triggered by MIS-C mimics KD. Kawasaki disease and MIS-C do share some 
similar clinical and laboratory features including conjunctival injection, rash, high levels of CRP. Differentiating between $\mathrm{KD}$ and a Kawasaki mimic MIS-C may be challenging. However, based on our experience and the relevant literature a number of points may be highlighted, clinically there are certain features that are more specific to MIS-C, which we do not encounter in KD: MIS-C patients often have gastrointestinal features including severe abdominal pain and myocardial involvement is prominent in MIS-C. As for the age, the MIS-C patients can have an older age of onset, whereas almost all of our KD patients are younger than 5 years of age. As to the lab results, MIS-C patients have lymphopenia, whereas the lymphocyte count is normal in KD. In MIS-C patients, proBNP and D-dimer levels are high, which again is not expected in KD. Finally, although CRP levels are high in KD as well and we cannot offer a cut-off, the CRP levels are much higher in MIS-C patients. Therefore, we believe that this report will be useful to understand actual clinical course of the MIS-C cases and may guide patient care.

As a conclusion, our study documents an accumulation of MIS-C cases in Turkey and its association with COVID-19. The MIS-C patients reported here had certain features that differ from those of COVID-19 patients with severe/critical disease course: some of the children who had this new emerging and life-threatening entity seems to present with predominant Kawasaki-like symptoms including longer fever duration and rash, bilateral conjunctival injection, peripheral ede$\mathrm{ma}$, and cardiac and acute gastrointestinal symptoms and altered mental status. When these clinical findings, particularly conjunctival injection and rash is supported by the higher acute phase response in some laboratory elements such as $\operatorname{WBC}(1,100 / \mu \mathrm{L})$ and $\mathrm{CRP}(>12.36 \mathrm{mg} / \mathrm{dl})$, the accurate diagnosis of MIS-C seems likely possible. Corticosteroids with IVIG and biologic immunomodulatory treatments such as anakinra seem promising for the management of MIS-C cases.

Author contributors' statement $\quad$ Dr. Ozsurekci, Dr. Ceyhan, Dr. Cengiz, and Dr. Ozen conceptualized and designed the study, collected data, drafted the initial manuscript, and reviewed and revised the manuscript.

Dr. Ozsurekci, Dr. Kesici, Dr. Akca, Dr. Gurlevik, Dr. Oygar, Dr. Ilbay, Dr. Aykac, Dr. Cura Yayla, Dr. Sartas Nakip, Dr. Karacanoglu, Dr. Katlan, Dr. Basaran, Dr. Bayrakci, and Dr. Bilginer carried out the initial analyses, collected data, and reviewed and revised the manuscript. Dr. Karakaya performed statistical analyses. Dr. Ozsurekci, Dr. Ceyhan, Dr. Ozen, and Dr. Cengiz critically reviewed the manuscript for important intellectual content. All authors approved the final manuscript as submitted and agree to be accountable for all aspects of the work.

Data availability statement The data that support the findings of this study are available on request from the corresponding author. The data are not publicly available due to privacy or ethical restrictions.

\section{Declarations}

Conflict of interest The other authors have indicated they have no potential conflicts of interest to disclose
Financial disclosure The authors have indicated they have no financial relationships relevant to this article to disclose.

\section{References}

1. Castagnoli R, Votto M, Licari A, Brambilla I, Bruno R, Perlini S, Rovida F, Baldanti F, Marseglia GL (2020) Severe acute respiratory syndrome coronavirus 2 (SARS-CoV-2) infection in children and adolescents: a systematic review. JAMA Pediatr 174:882-889

2. Rodriguez-Morales AJ, Cardona-Ospina JA, Gutierrez-Ocampo E et al (2020) Clinical, laboratory and imaging features of COVID19: a systematic review and meta-analysis. Travel Med Infect Dis 34:101623

3. Ludvigsson JF (2020) Systematic review of COVID-19 in children shows milder cases and a better prognosis than adults. Acta Paediatr 109:1088-1095

4. Lu X, Zhang L, Du H et al (2020) SARS-CoV-2 infection in children. N Engl J Med 382:1663-1665

5. Cura Yayla BC, Ozsurekci Y, Aykac K et al (2020) Characteristics and management of children with COVID-19 in Turkey. Balkan Med J 37:341-347

6. Riphagen S, Gomez X, Gonzales-Martinez C et al (2020) Hyperinflammatory shock in children during COVID-19 pandemic. Lancet 395(10237):1607-1608

7. Centers for Disease Control and Prevention (2020) Emergency preparedness and response: health alert network. https:/emergency. cdc.gov/han/2020/han00432.asp. Accessed Nov 102020

8. World Health Organization. Multisystem inflammatory syndrome in children and adolescents with COVID-19 (2020) www.who.int/ publications-detail/multisystem-inflammatory-syndrome-inchildren-and-adolescents-with-covid-19. Accessed November 10, 2020

9. Consiglio CR, Cotugno N, Sardh F et al (2020) The immunology of multisystem inflammatory syndrome in children with COVID-19. Cell 183(4):968-981.e7

10. Ahmed M, Advani S, Moreira A, Zoretic S, Martinez J, Chorath K, Acosta S, Naqvi R, Burmeister-Morton F, Burmeister F, Tarriela A, Petershack M, Evans M, Hoang A, Rajasekaran K, Ahuja S, Moreira A (2020) Multisystem inflammatory syndrome in children: a systematic review. EClinicalMedicine 26:100527

11. Toubiana J, Poirault C, Corsia A et al (2020) Kawasaki-like multisystem inflammatory syndrome in children during COVID-19 pandemic in Paris, France: prospective observational study. BMJ 369: $\mathrm{m} 2094$

12. Akca UK, Kesici S, Ozsurekci Y, Aykan HH, Batu ED, Atalay E, Demir S, Sag E, Vuralli D, Bayrakci B, Bilginer Y, Ozen S (2020) Kawasaki-like disease in children with COVID-19. Rheumatol Int 40:2105-2115

13. Dong Y, Mo X, Hu Y et al (2020) Epidemiological characteristics of 2143 pediatric patients with 2019 coronavirus disease in China. Pediatrics 58(4):712-713

14. Shen KL, Yang YH, Jiang RM et al (2020) Updated diagnosis, treatment and prevention of COVID-19 in children: experts' consensus statement (condensed version of the second edition). World J Pediatr 24:1-8

15. Carter MJ, Fish M, Jennings A, Doores KJ, Wellman P, Seow J, Acors S, Graham C, Timms E, Kenny J, Neil S, Malim MH, Tibby SM, Shankar-Hari M (2020) Peripheral immunophenotypes in children with multisystem inflammatory syndrome associated with SARS-CoV-2 infection. Nat Med 26(11):1701-1707

16. Belhadjer Z, Meot M, Bajolle F et al (2020) Acute heart failure in multisystem inflammatory syndrome in children (MIS-C) in the context of global SARS-CoV-2 pandemic. Circulation 142(5): $429-436$ 
17. Mathew D, Giles JR, Baxter AE et al (2020) Deep immune profiling of COVID-19 patients reveals distinct immunotypes with therapeutic implications. Science 369:eabc8511

18. Laing AG, Lorenc A, Del Barrio IM et al (2020) A consensus COVID-19 immune signature combines immune-protection with discrete sepsis-like traits associated with poor prognosis. Preprint at medRxiv

19. Cheung EW, Zacharian P, Gorelik M et al (2020) Multisystem inflammatory syndrome related to COVID-19 in previously healthy children and adolescents in New York City. JAMA 324:294-296

20. Moraleda C, Serna-Pascual M, Soriano-Arandes A et al (2020) Multi-inflammatory syndrome in children related to SARS-CoV-2 in Spain. Clin Infect Dis 25:ciaa1042

21. Verdoni L, Mazza A, Gervasoni et al (2020) An outbreak of severe Kawasaki-like disease at the Italian epicentre of the SARS-CoV-2 epidemic: an observational cohort study. Lancet 395:1771-1778

22. Whittaker E, Bamford A, Kenny J, Kaforou M, Jones CE, Shah P, Ramnarayan P, Fraisse A, Miller O, Davies P, Kucera F, Brierley J, McDougall M, Carter M, Tremoulet A, Shimizu C, Herberg J, Burns JC, Lyall H, Levin M, for the PIMS-TS Study Group and EUCLIDS and PERFORM Consortia (2020) Clinical characteristics of 58 children with a pediatric inflammatory multisystem syndrome temporally associated with SARS-Cov-2. JAMA 324:259269

23. Dufort EM, Koumans EH, Chow EJ, Rosenthal EM, Muse A, Rowlands J, Barranco MA, Maxted AM, Rosenberg ES, Easton D, Udo T, Kumar J, Pulver W, Smith L, Hutton B, Blog D, Zucker H, New York State and Centers for Disease Control and Prevention Multisystem Inflammatory Syndrome in Children Investigation Team (2020) Multisystem inflammatory syndrome in children and adolescents in New York City. N Engl J Med 383:347-358

24. Chiotos K, Bassiri H, Behrens EM (2020) Multisystem inflammatory syndrome in children during the coronavirus 2019 pandemic: a case series. J Pediatric Infect Dis Soc 9:393-398

25. Jones VG, Mills M, Suarez D (2020) COVID-19 and Kawasaki disease: novel virus and novel case. Hosp Pediatr 10:537-540

26. Waltuch T, Gill P, Zinns LE (2020) Features of COVID-19 postinfectious cytokine release syndrome in children presenting to the emergency department. Am J Emerg Med 38(10):2246.e3-2246.e6

27. Dolhnikoff M, Ferranti JF, Monteiro RAA et al (2020) SARS-CoV2 in cardiac tissue of a child with COVID-19-related multisystem inflammatory syndrome. Lancet 4:790-794

28. Kazatchkine MD, Kaveri SV, Kazatchkine MD et al (2001) Immunomodulation of autoimmune and inflammatory diseases with intravenous immune globulin. N Engl J Med 345:747-755

29. Feldstein LR, Rose EB, Horwitz SM et al (2020) Multisystem inflammatory syndrome in U.S. children and adolescents. N Engl $\mathrm{J}$ Med 383(4):334-346

30. Godfred-Cato S, Bryant B, Leung J, Oster ME, Conklin L, Abrams J, Roguski K, Wallace B, Prezzato E, Koumans EH, Lee EH, Geevarughese A, Lash MK, Reilly KH, Pulver WP, Thomas D, Feder KA, Hsu KK, Plipat N, Richardson G, Reid H, Lim S, Schmitz A, Pierce T, Hrapcak S, Datta D, Morris SB, Clarke K, Belay E, California MIS-C Response Team (2020) COVID-19associated multisystem inflammatory syndrome in children-
United States, March-July 2020. MMWR Morb Mortal Wkly Rep 69:1074-1080

31. Pouletty M, Borocco C, Ouldali N, Caseris M, Basmaci R, Lachaume N, Bensaid P, Pichard S, Kouider H, Morelle G, Craiu I, Pondarre C, Deho A, Maroni A, Oualha M, Amoura Z, Haroche J, Chommeloux J, Bajolle F, Beyler C, Bonacorsi S, Carcelain G, Koné-Paut I, Bader-Meunier B, Faye A, Meinzer U, Galeotti C, Melki I (2020) Paediatric multisystem inflammatory syndrome temporally associated with SARS-CoV-2 mimicking Kawasaki disease (Kawa-COVID-19): a multicentre cohort. Ann Rheum Dis 79:999-1006

32. Morris SB, Schwartz NG, Patel P et al (2020) Case series of multisystem inflammatory syndrome in adults associated with SARSCoV-2 infection-United Kingdom and United States, MarchAugust 2020. MMWR 69(40):1450-1456

33. Gupta A, Madhavan MV, Sehgal K, Nair N, Mahajan S, Sehrawat TS, Bikdeli B, Ahluwalia N, Ausiello JC, Wan EY, Freedberg DE, Kirtane AJ, Parikh SA, Maurer MS, Nordvig AS, Accili D, Bathon JM, Mohan S, Bauer KA, Leon MB, Krumholz HM, Uriel N, Mehra MR, Elkind MSV, Stone GW, Schwartz A, Ho DD, Bilezikian JP, Landry DW (2020) Extrapulmonary manifestations of COVID-19. Nat Med 26:1017-1032

34. Rhen T, Cidlowski JA (2005) Antiinflammatory action of glucocorticoids-new mechanism for old drugs. N Engl J Med 353:1711-1723

35. Cao B, Gao H, Zhou B, Deng X, Hu C, Deng C, Lu H, Li Y, Gan J, Liu J, Li H, Zhang Y, Yang Y, Fang Q, Shen Y, Gu Q, Zhou X, Zhao W, Pu Z, Chen L, Sun B, Liu X, Hamilton CD, Li L (2016) Adjuvant corticosteroid treatment in adults with influenza A (H7N9) viral pneumonia. Crit Care Med 44:e318-e328

36. Shang L, Zhao J, Hu Y, du R, Cao B (2020) On the use of corticosteroids for 2019-nCoV pneumonia. Lancet 395:683-684

37. Chen RC, Tang XP, Tan SY, Liang BL, Wan ZY, Fang JQ, Zhong N (2006) Treatment of severe acute respiratory syndrome with glucosteroids: the Guangzhou experience. Chest 129:1441-1452

38. Li H, Yang SG, Gu L, Zhang Y, Yan XX, Liang ZA, Zhang W, Jia HY, Chen W, Liu M, Yu KJ, Xue CX, Hu K, Zou Q, Li LJ, Cao B, Wang $C$, the National Influenza A(H1N1)pdm09 Clinical Investigation Group of China (2017) National Influenza A(H1N1)pdm09 Clinical Investigation Group of China, Effect of low-to-moderate-dose corticosteroids on mortality of hospitalized adolescents and adults with influenza A(H1N1)pdm09 viral pneumonia, Influenza. Other Respir Viruses 11:345-354

39. Siemieniuk RAC, Meade MO, Alonso-Coello P, Briel M, Evaniew N, Prasad M, Alexander PE, Fei Y, Vandvik PO, Loeb M, Guyatt GH (2015) Corticosteroid therapy for patients hospitalized with community-acquired pneumonia: a systematic review and metaanalysis. Ann Intern Med 163:519-528

40. Buckley LF, Wohlford GF, Ting C et al (2020) Role for anticytokine therapies in severe coronavirus disease 2019. Crit Care Expl 2:e0178

Publisher's note Springer Nature remains neutral with regard to jurisdictional claims in published maps and institutional affiliations. 